\title{
Electronic Friction Dominates Hydrogen Hot-Atom Relaxation on Pd(100)
}

\author{
M. Blanco-Rey, ${ }^{1,2}$ J. I. Juaristi, ${ }^{1,3,2}$ R. Díez Muiño, ${ }^{3,2}$ H. F. Busnengo, ${ }^{4}$ G. J. Kroes, ${ }^{5}$ and M. Alducin ${ }^{3,2}$ \\ ${ }^{1}$ Departamento de Física de Materiales, Facultad de Químicas UPV/EHU, Apartado 1072, 20018 Donostia-San Sebastián, Spain \\ ${ }^{2}$ Donostia International Physics Center (DIPC), Paseo Manuel de Lardizabal 4, 20018 Donostia-San Sebastián, Spain \\ ${ }^{3}$ Centro de Física de Materiales CFM/MPC (CSIC-UPV/EHU), Paseo Manuel de Lardizabal 5, 20018 Donostia-San Sebastián, Spain \\ ${ }^{4}$ Instituto de Física Rosario and Universidad Nacional de Rosario, 2000 Rosario, Argentina \\ ${ }^{5}$ Leiden Institute of Chemistry, Gorlaeus Laboratories, Leiden University, P.O. Box 9502, 2300 RA Leiden, The Netherlands
}

(Received 13 December 2013; revised manuscript received 6 February 2014; published 14 March 2014)

\begin{abstract}
We study the dynamics of transient hot $\mathrm{H}$ atoms on $\mathrm{Pd}(100)$ that originated from dissociative adsorption of $\mathrm{H}_{2}$. The methodology developed here, denoted AIMDEF, consists of $a b$ initio molecular dynamics simulations that include a friction force to account for the energy transfer to the electronic system. We find that the excitation of electron-hole pairs is the main channel for energy dissipation, which happens at a rate that is five times faster than energy transfer into Pd lattice motion. Our results show that electronic excitations may constitute the dominant dissipation channel in the relaxation of hot atoms on surfaces.
\end{abstract}

Electron-hole $(e-h)$ pair excitations are an unquestioned efficient energy drain in the interaction of fast atoms with solids and surfaces [1-4]. In contrast, the relevance of this dissipation channel in gas-surface interactions that involve energies up to a few $\mathrm{eV}$ is not so clear. It depends not only on the specific system, but also on the elementary process considered, as shown by different studies on scattering [5-12] and adsorption [6,13-26] of atoms and molecules on surfaces. Low-energy $e$ - $h$ pair excitations have been detected as chemicurrents on Schottky diode devices during the chemisorption of atomic and molecular species on metals [13-15]. The correlation found between chemicurrent intensities and adsorption energies is a strong indication that a large fraction of the energy dissipated in both the dissociative and nondissociative adsorption processes is used to excite $e-h$ pairs. However, this strongly contrasts with examples showing that the dissociative adsorption is reasonably well described within the electronically adiabatic approach, which neglects the coupling between electronic excitations and the nuclear motion [6,18-20,23,25], and that the effect of electronic energy dissipation seems negligible $[21,24,26]$. Therefore, a question that is raised here is at what stage of the dissociative adsorption process $e-h$ pair excitations do become relevant.

In a typical adsorption event, the incoming gas species gain additional kinetic energy when entering the attractive adsorption well. In the particular case of dissociative adsorption, this energy gain can lead to the formation of so-called "hot" atoms or fragments, with energies much larger than the corresponding thermal energies of the substrate atoms. The formed hot species will then propagate along the surface until they dissipate the excess kinetic energy and finally accommodate at a stable adsorption position. This stage of the dissociative adsorption process, where the relevance of $e-h$ pair excitations has been traditionally neglected, is the focus of the present work.
In this Letter we show that while $e-h$ pair excitation may not be relevant on the molecule-bond-breaking time scale, it is an efficient dissipative channel in the subsequent relaxation of the resulting transient hot products. Our finding provides a new perspective for the study of hot atom (HA) relaxation on metal surfaces, which hitherto focused mainly on the energy transfer to lattice vibrations [27-29]. The traveled mean free paths following dissociative adsorption may determine, for example, the likelihood of HA recombination with adsorbed species. Since those distances are related to the HA energy dissipation rate, the competition between dissipation channels is a key factor that affects the surface reactivity. We have chosen dissociative adsorption of $\mathrm{H}_{2}$ on $\operatorname{Pd}(100)$ as a case study. Previous theoretical work on this system was carried out by Gross using ab initio molecular dynamics (AIMD) [29]. According to this study each HA is relaxed by transferring kinetic energy to the Pd atoms at a rather fast rate: In $800 \mathrm{fs}$ the $\mathrm{H}$ atoms lose $50 \%$ of the energy acquired upon dissociation. Here we demonstrate that for $\mathrm{H}_{2}$ molecules impinging at similar kinetic energies the relaxation rate by means of $e-h$ pair excitations is in fact much faster. The incipient HAs need only 150 fs to reduce their initial kinetic energy by half. This implies that energy dissipation into electronic excitations occurs faster, by a factor of 5, than into lattice vibrations.

The effect of low-energy $e-h$ pair excitations can be effectively introduced in the classical equations of motion through a friction force that accounts for energy dissipation $[21,30]$. The technique we use here, which we denote AIMDEF (AIMD with electronic friction), consists of AIMD simulations that include and calculate the electronic friction force on the fly. This friction force acting on the gas-phase atom, $-\eta\left(\mathbf{r}_{i}\right) \dot{\mathbf{r}}_{i}$, is evaluated in the local density friction approximation (LDFA) [21]. The latter uses, at each time step, the friction coefficient $\eta\left(\mathbf{r}_{i}\right)$ of the atom 
embedded in a homogeneous free electron gas [31-33] with density equal to the $a b$ initio electron density of the bare surface at the position $\mathbf{r}_{i}$ of the gas-phase atom. This methodology, which we have implemented in the VASP package based on density functional theory (DFT) [34], is used here for the first time in combination with AIMD. The integration of these two tools into AIMDEF is very well suited to study dynamical processes in which energy dissipation to phonons and $e-h$ pairs are competing processes. This is the case for the example of HA propagation studied here.

AIMDEF simulations are carried out for individual $\mathrm{H}$ hot atoms in the frozen surface (FS) approach and also in the non-frozen surface (NFS) approach, in which the Pd atoms of the two outermost layers are allowed to move. In the NFS approach, the friction forces are also calculated for the undistorted surface electron density. The use of this approximation is justified, since the relevant electron density values are not expected to change significantly upon the Pd displacements present in our simulations. The initial conditions for the $\mathrm{H}$ atoms are taken from adiabatic frozen surface molecular dynamics (MD) calculations of $\mathrm{H}_{2}$ dissociation on $\mathrm{Pd}(100)$. The simulations were performed on a precalculated six-dimensional potential energy surface (PES) that was constructed by Lozano et al. from DFT data using structural and electronic settings similar to the ones used in the present Letter [35]. In those MD simulations a molecule was considered to be fully dissociated (and thus the trajectory terminated) when the $\mathrm{H}-\mathrm{H}$ distance reached three times the equilibrium gas-phase $\mathrm{H}_{2}$ bond length. The final coordinates and velocities, $\left(x, y, z ; v_{x}, v_{y}, v_{z}\right)$, of each $\mathrm{H}$ atom are used here as initial conditions in the AIMDEF calculations. The stopping criterion for the FS simulations is that the total energy reaches that of the $\mathrm{H}$ adsorbed at the hollow site plus its zero point energy. The present use of single- $\mathrm{H}-$ atom simulations to represent the full thermalization of the dissociating $\mathrm{H}_{2}$ is well justified in this system. As found in Ref. [29], when both $\mathrm{H}$ atoms are allowed in the simulation unit cell the trajectory details may change, but the average values of the quantities of interest, such as energies and traveled distances, remain unaffected.

Energies and forces have been evaluated from DFT with projector-augmented wave pseudopotentials [36] and a plane-wave basis set [34], using the supercell approach [37] and the Perdew-Wang 1991 generalized gradient approximation for the exchange and correlation functional [38]. AIMDEF simulations are run for individual $\mathrm{H}$ atoms on the $(2 \times 2)$ supercell. The equations of motion have been integrated with a small time step $h=0.1 \mathrm{fs}$ using Beeman's method, which is a predictor-corrector method for velocities. The velocities predicted at each step are used to evaluate the friction force. The error in the corrected velocities in the implemented Beeman's method is $O\left(h^{3}\right)$, i.e., smaller than the error $O\left(h^{2}\right)$ given by the usual velocity
Verlet scheme $[39,40]$. The latter may not be stable enough in the presence of friction forces.

The results reported below are obtained for $\mathrm{H}_{2}$ molecules impinging on the $\operatorname{Pd}(100)$ surface in their rovibrational ground state at normal incidence with kinetic energy $E_{i}=$ 0.5 and $1.0 \mathrm{eV}$. In total we have analyzed 182(178) AIMDEF atomic trajectories that correspond to 91(89) dissociating molecules from an initial sampling of $100 \mathrm{H}_{2}$ trajectories for $E_{i}=0.5(1.0) \mathrm{eV}$. These sets of trajectories constitute statistically representative samples of the dissociated molecules [41]. Except for one trajectory (three trajectories) in which penetration of the subsurface occurs for $E_{i}=0.5(1.0) \mathrm{eV}$, all the $\mathrm{H}$ atoms remain on the surface. In order to have a benchmark for the relaxation times in the absence of $e-h$ pair excitations, we have first carried out NFS AIMD simulations without electronic friction on 100 atomic trajectories for $E_{i}=0.5 \mathrm{eV}$ and $h=1 \mathrm{fs}$, in a similar fashion as done by Gross [29]. This choice of time step ensures that the total energy drift is $\lesssim 20 \mathrm{meV}$. The conversion of $\mathrm{H}_{2}$ kinetic energy into $\mathrm{Pd}$ atom motion as a function of time, which is calculated as an average over the available trajectories, is shown in the inset of Fig. 1. The averaged starting kinetic energy for each pair of $\mathrm{H}$ atoms in the AIMDEF simulations is $\simeq 0.8 \mathrm{eV}$. The latter value amounts to the initial molecular incident $E_{i}$ plus the energy gained by the interaction with the surface, as already noted in Ref. [29]. The kinetic energy loss rate is in good agreement with the AIMD results reported by Gross. The small differences at long times are caused by the use of different initial conditions, cell sizes, and number of mobile Pd layers.

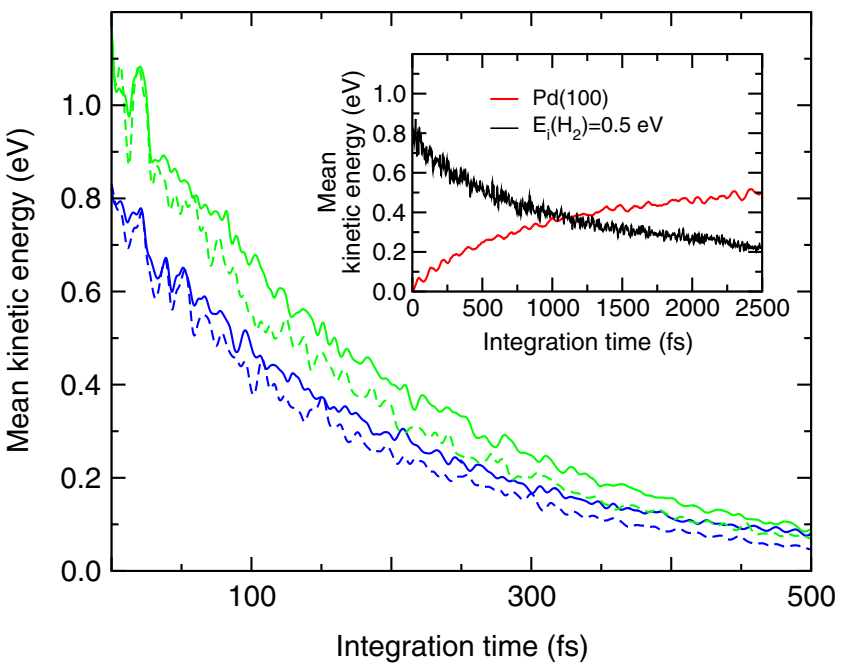

FIG. 1 (color online). Main panel: mean $\mathrm{H}_{2}$ kinetic kinetic energy as a function of time. Blue (green) lines correspond to molecules impinging with $E_{i}=0.5(1.0) \mathrm{eV}$. Solid (dashed) lines represent the AIMDEF results obtained in the FS (NFS) approach. Inset: kinetic energy in the absence of electronic friction as a function of time. The energy lost by $\mathrm{H}_{2}$ (black line) is transferred into $\mathrm{Pd}$ atom motion (red line). 
The prominent role of $e-h$ pair excitations in the energy dissipation of the formed HAs becomes evident by comparing the results of the inset with the ones we obtain from the AIMDEF simulations. Figure 1 shows the mean kinetic energy as a function of time including the effect of electronic friction by AIMDEF. For $E_{i}=0.5 \mathrm{eV}$, the $\mathrm{H}$ atoms lose $50 \%$ of their energy into $e$ - $h$ pair excitations in just $150 \mathrm{fs}$, as shown by the exponentially decaying FS curve, while they need 800 fs to dissipate the same amount of energy into $\mathrm{Pd}$ atom motion if no $e-h$ pair excitations are allowed (see the inset). The finding that the transient products of dissociative adsorption are relaxed by $e-h$ pair excitations much more efficiently than by substrate vibrations constitutes the main result of the present Letter. The kinetic energy decay rate of faster impinging molecules with $E_{i}=1.0 \mathrm{eV}$ almost coincides with that for $E_{i}=0.5 \mathrm{eV}$. This implies that similar electron densities are being experienced by the HAs in both cases, as explained below. The NFS curves, which combine both loss channels, lie close to the FS ones, confirming that the electronic mechanism for energy loss dominates over the energy transfer to the Pd lattice. The similar decay found in the FS and NFS cases is reasonable in view of the largely different time scales of both dissipation mechanisms. The remarkable outcome of the present case study is that, even though $e-h$ pair excitations may often be neglected in the calculation of dissociation probabilities [21,24,26], most of the energy of the dissociation products is transferred to the electronic system. Importantly, this is consistent with the fact that chemicurrent intensities measured during the chemisorption of atoms and molecules scale with the adsorption energy [13-15]. Chemicurrents have also been observed during the associative desorption of $\mathrm{H}_{2}$ on metal surfaces $[42,43]$. Our finding shows that in these experiments a large amount of $e-h$ pair excitation may take place during the motion of the $\mathrm{H}$ atoms on the surface prior to recombination.

It is meaningful to analyze our electronic dissipation rates in terms of the surface electronic densities that the $\mathrm{H}$ atoms experience during the thermalization process. Analyzing our AIMDEF calculations, we find that, on average, the mean electron radius probed by the $\mathrm{H}$ atoms along their trajectories is $\left\langle r_{s}\right\rangle \sim 2.75$ a.u., where $r_{s}$ is defined in terms of the electron density as $r_{s}=$ $\left[3 /\left(4 \pi n_{0}\right)\right]^{1 / 3}$. According to the LDFA [21], the electronic friction coefficient that corresponds to this mean radius of the free electron gas is $\langle\eta\rangle=0.18$ a.u.. As a first approximation, one might attempt to extract this value from the exponential decay observed in Fig. 1. Thus, if we disregard the PES topography and consider a constant potential model, the energy loss follows a simple exponential decay with rate $\lambda=-2 \tilde{\eta} / m_{p}$, where $m_{p}$ is the proton mass and $\tilde{\eta}$ is an effective average friction coefficient. A fit of the FS curves shown in Fig. 1 to this model results in $\tilde{\eta}=$ 0.111 a.u. for both incidence energies. In the LDFA
[21], this value of the friction coefficient arises for an effective mean radius of the free electron gas $\tilde{r}_{s}=3.75$ a.u., i.e., a value that corresponds to an electron density smaller than the actual average electron density probed by the $\mathrm{H}$ atoms $\left(\left\langle r_{s}\right\rangle \sim 2.75\right.$ a.u. $)$.

The difference between the actual average value of the friction coefficient, $\langle\eta\rangle$, and the one extracted from the FS curves, $\tilde{\eta}$, can be understood with the help of toy models in terms of the PES corrugation. In the region where the HAs move, the potential along the direction normal to the surface $z$ can be reasonably approximated by a harmonic potential. For such a model potential, the equation of motion in the $z$ coordinate corresponds to that of a damped harmonic oscillator. Hence, the energy decay rate is $\lambda=-\tilde{\eta} / m_{p}$; i.e., it is slower than the decay rate of the constant potential model. A similar conclusion can be obtained for the corrugation along the coordinates parallel to the surface $(x, y)$. Considering for simplicity a one-dimensional scenario with a sine potential $V(x)=V_{0} \sin (a x)$, it can be shown that the decay is also slower for increasing corrugation values, $V_{0}$. Therefore, generalizations to other surfaces and HA species considering just the typical electronic density values of the specific metal will result in coarse estimates of HA relaxation rates by $e-h$ pair excitations. Obtaining realistic values requires more complex modeling, as the actual rates are highly dependent on the system details. AIMDEF comes in as a valuable tool for this task.

The discussion above accounts for the $e$ - $h$ pair excitation effect on the HA relaxation time scale. The effect on the length scale, i.e., on the distances traveled by the HA, is discussed next for the FS case. The total traveled path length, denoted $L$ in the inset of Fig. 2, is directly connected to the energy loss by electronic friction. $L$ depends substantially on the initial energy, as shown in the distributions of Fig. 2. For $E_{i}=0.5 \mathrm{eV}$, the distribution is peaked at $\simeq 25 \AA$ and has a width of $20 \AA$, while the distribution for $E_{i}=1.0 \mathrm{eV}$ is peaked at $\gtrsim 30 \AA$ and is narrower. Note, however, that information on the total path length cannot be directly extracted from experiments and other magnitudes should be considered instead.

Scanning tunneling microscopy (STM) has been used in other systems to obtain the final lateral mean distance between dissociation products [44-48], denoted $D$ in the inset of Fig. 2. Information on the lateral displacement of an individual atom after dissociation, i.e., the in-plane distance between its final and initial position (denoted $d$ in the inset of Fig. 2), can also be measured when using the STM to induce single-molecule manipulations [49,50]. Thus, we have also analyzed the $d$ - and $D$-distributions of the relaxed $\mathrm{H}$ atoms. The results are shown in Fig. 3. In both cases the effect of the incident energy is very subtle, in contrast with our finding for the path length distributions. For the studied $E_{i}$ values most atoms are displaced by $d \lesssim 5 \AA$. The distributions of $D$ values are widely spread. Dissipation 


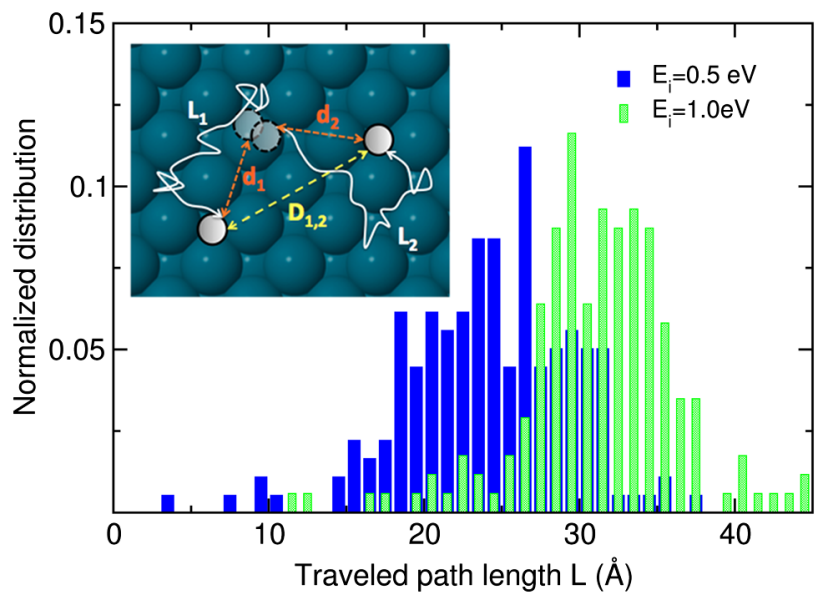

FIG. 2 (color online). Inset: sketch of the distances traveled by the $\mathrm{H}$ atoms after dissociation. Small blue and white circles show the initial and final positions of the $\mathrm{H}$ atoms, respectively. Main panel: distributions of the total length of the pathways traveled by the $\mathrm{H}$ atoms after dissociation in the FS case, depicted as solid white lines $\left(L_{1,2}\right)$ in the inset.

by electronic friction results in an average $\mathrm{H}-\mathrm{H}$ lateral distance $\langle D\rangle=5.76 \AA$ for $E_{i}=0.5 \mathrm{eV}$, a factor of 2 smaller than the value $\langle D\rangle \sim 12 \AA$ found in the AIMD simulations without friction [29]. The latter is consistent with the more efficient energy dissipation provided by the electronic channel. However, it is difficult to establish a direct comparison between the electronic and phononic energy dissipation rates and the corresponding lateral distances $D$. As sketched in the inset of Fig. 2, the HAs typically follow a rather erratic walk that makes it difficult to relate the total length $L$ with $D$. In particular, since the HAs do not diffuse thermally, results known from random walk models cannot be applied here. More importantly, notice that an estimate of the friction coefficient made from the lateral distances observed by STM could provide a largely overestimated value, as proven by our finding that $D, d \ll L$ due to the aforementioned erratic trajectories.

In summary, we have performed $a b$ initio molecular dynamics simulations that incorporate on the fly the effect of $e-h$ pair excitations by means of a friction force that is calculated within the local density friction approximation [21]. Using this methodology, which we denote AIMDEF, we have shown that for $\mathrm{H}_{2}$ molecules dissociating on $\operatorname{Pd}(100)$ the transient $\mathrm{H}$ atoms propagate nonthermally on the surface and rapidly lose energy into substrate $e$ - $h$ pair excitations. Only $150 \mathrm{fs}$ are necessary for the kinetic energy of the incipient $\mathrm{H}$ hot atoms to be reduced by half. Interestingly, this energy loss rate is five times faster than that due to the surface vibration channel [29] and leads to the $\mathrm{H}$ atoms being settled at the adsorption sites on a timescale of few hundred femtoseconds. The efficiency of the electronic dissipation channel is a consequence of the time spent by the $\mathrm{H}$ atoms in surface regions of substantial electron density $\left(r_{s}=2-3\right.$ a.u. $)$. Given that these
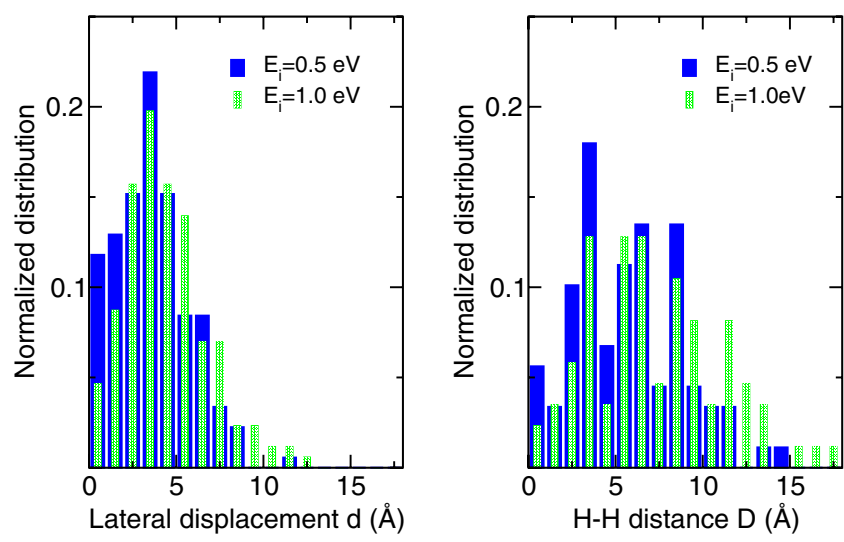

FIG. 3 (color online). Left hand panel: distribution of in-plane displacement of $\mathrm{H}$ atoms after dissociation in the FS case. Right hand panel: distribution of in-plane $\mathrm{H}-\mathrm{H}$ distance after dissociation. The quantities shown in these panels correspond to distances $d$ and $D$ in the inset of Fig. 2 .

conditions are inherent in the relaxation of hot atoms and molecules on metals, our study shows that dissipation into $e$ - $h$ pairs cannot be neglected and should be considered on the same footing as dissipation into lattice vibrations. However, generalizations to other atoms and substrates on the relative relevance of each dissipation channel are not straightforward, as the atom mass and PES topography are key factors to be considered. In this respect, the proposed AIMDEF method is a valuable methodology because it gives an accurate account of the system PES and it models both energy dissipation channels simultaneously. All in all, we have shown that although $e$ - $h$ pair excitations may not have much influence on whether an impinging molecule dissociates, they can have a major impact on the subsequent relaxation of the products.

This work has been supported in part by the Basque Departamento de Educación, Universidades e Investigación, the University of the Basque Country UPV/EHU (Grant No. IT-756-13) and the Spanish Ministerio de Ciencia e Innovación (Grant No. FIS201019609-C02-02). M. B.-R. acknowledges financial support from the Gipuzkoako Foru Aldundia and the European Commission (Grant No. FP7-PEOPLE-2010-RG276921) and G. J. K. from CW/NWO TOP grant. Computational resources were provided by the DIPC computing center.

[1] H. Winter, Phys. Rep. 367, 387 (2002).

[2] H. Winter, J. I. Juaristi, I. Nagy, A. Arnau, and P. M. Echenique, Phys. Rev. B 67, 245401 (2003).

[3] M. A. Zeb, J. Kohanoff, D. Sánchez-Portal, A. Arnau, J. I. Juaristi, and E. Artacho, Phys. Rev. Lett. 108, 225504 (2012).

[4] D. Goebl, K. Khalal-Kouache, D. Roth, E. Steinbauer, and P. Bauer, Phys. Rev. A 88, 032901 (2013). 
[5] J. D. White, J. Chen, D. Matsiev, D. J. Auerbach, and A. M. Wodtke, Nature (London) 433, 503 (2005).

[6] P. Nieto, E. Pijper, D. Barredo, G. Laurent, R. A. Olsen, E.-J. Baerends, G.-J. Kroes, and D. Farías, Science 312, 86 (2006).

[7] G.-J. Kroes, Science 321, 794 (2008).

[8] N. Shenvi, S. Roy, and J. C. Tully, Science 326, 829 (2009).

[9] S. Monturet and P. Saalfrank, Phys. Rev. B 82, 075404 (2010).

[10] L. Martin-Gondre, M. Alducin, G. A. Bocan, R. Díez Muiño, and J. I. Juaristi, Phys. Rev. Lett. 108, 096101 (2012).

[11] M. Pavanello, D. J. Auerbach, A. M. Wodtke, M. BlancoRey, M. Alducin, and G.-J. Kroes, J. Phys. Chem. Lett. 4, 3735 (2013).

[12] N. Bartels, K. Golibrzuch, C. Bartels, L. Chen, D. J. Auerbach, A. M. Wodtke, and T. Schäfer, Proc. Natl. Acad. Sci. U.S.A. 110, 17738 (2013).

[13] B. Gergen, H. Nienhaus, W. H. Weinberg, and E. W. McFarland, Science 294, 2521 (2001).

[14] H. Nienhaus, H. Bergh, B. Gergen, A. Majumdar, W. Weinberg, and E. McFarland, Surf. Sci. 445, 335 (2000).

[15] H. Nienhaus, Surf. Sci. Rep. 45, 1 (2002).

[16] J. R. Trail, M. C. Graham, D. M. Bird, M. Persson, and S. Holloway, Phys. Rev. Lett. 88, 166802 (2002).

[17] M. Lindenblatt, and E. Pehlke, Phys. Rev. Lett. 97, 216101 (2006).

[18] C. Díaz, J. K. Vincent, G. P. Krishnamohan, R. A. Olsen, G. J. Kroes, K. Honkala, and J. K. Nørskov, Phys. Rev. Lett. 96, 096102 (2006).

[19] M. Alducin, R. Díez Muiño, H. F. Busnengo, and A. Salin, Phys. Rev. Lett. 97, 056102 (2006).

[20] A. Salin, J. Chem. Phys. 124, 104704 (2006).

[21] J. I. Juaristi, M. Alducin, R. Díez Muiño, H. F. Busnengo, and A. Salin, Phys. Rev. Lett. 100, 116102 (2008).

[22] M. Timmer and P. Kratzer, Phys. Rev. B 79, 165407 (2009).

[23] C. Díaz, E. Pijper, R. A. Olsen, H. F. Busnengo, D. Auerbach, and G.-J. Kroes, Science 326, 832 (2009).

[24] J. Meyer and K. Reuter, New J. Phys. 13, 085010 (2011).

[25] I. Goikoetxea, J. Beltrán, J. Meyer, J. I. Juaristi, M. Alducin, and K. Reuter, New J. Phys. 14, 013050 (2012).

[26] G. Füchsel, S. Schimka, and P. Saalfrank, J. Phys. Chem. A 117, 8761 (2013).

[27] D. V. Shalashilin and B. Jackson, J. Chem. Phys. 109, 2856 (1998).

[28] N. Pineau, H. F. Busnengo, J. C. Rayez, and A. Salin, J. Chem. Phys. 122, 214705 (2005).

[29] A. Gross, Phys. Rev. Lett. 103, 246101 (2009).
[30] M. Head-Gordon and J. C. Tully, J. Chem. Phys. 103, 10137 (1995)

[31] P. M. Echenique, R. M. Nieminen, and R. H. Ritchie, Solid State Commun. 37, 779 (1981).

[32] P. M. Echenique, R. M. Nieminen, J. C. Ashley, and R. H. Ritchie, Phys. Rev. A 33, 897 (1986).

[33] A. Salin, A. Arnau, P. M. Echenique, and E. Zaremba, Phys. Rev. B 59, 2537 (1999).

[34] G. Kresse and J. Hafner, Phys. Rev. B 47, 558 (1993).

[35] A. Lozano, A. Gross, and H. F. Busnengo, Phys. Chem. Chem. Phys. 11, 5814 (2009).

[36] P. E. Blöchl, Phys. Rev. B 50, 17953 (1994).

[37] Our model Pd(100) surface consists of a five-layer $(2 \times 2)$ periodic slab and a vacuum region equivalent to nine interlayer spacings. The reciprocal space has been sampled with a $6 \times 6 \times 1$ Monkhorst-Pack mesh [51] and an energy cutoff of $350 \mathrm{eV}$. Electronic wave functions were converged with an energy tolerance $10^{-5} \mathrm{eV}$ and the two topmost layer spacings were relaxed by minimizing forces and total energies below $0.01 \mathrm{eV} / \AA$ and $10^{-4} \mathrm{eV}$, respectively.

[38] J. P. Perdew, J. A. Chevary, S. H. Vosko, K. A. Jackson, M. R. Pederson, D. J. Singh, and C. Fiolhais, Phys. Rev. B 46, 6671 (1992).

[39] P. Schofield, Comput. Phys. Commun. 5, 17 (1973).

[40] D. Beeman, J. Comput. Phys. 20, 130 (1976).

[41] The analysis of 5000 molecular trajectories that were generated by performing MD simulations on the six-dimensional PES shows that the selected set yields a good sampling of the $\mathrm{H}$ initial conditions and reproduces the sticking probability of $\sim 90 \%$.

[42] B. Schindler, D. Diesing, and E. Hasselbrink, J. Chem. Phys. 134, 034705 (2011).

[43] B. Schindler, D. Diesing, and E. Hasselbrink, J. Phys. Chem. C 117, 6337 (2013).

[44] H. Brune, J. Wintterlin, R. J. Behm, and G. Ertl, Phys. Rev. Lett. 68, 624 (1992).

[45] J. Wintterlin, R. Schuster, and G. Ertl, Phys. Rev. Lett. 77, 123 (1996).

[46] M. Schmid, G. Leonardelli, R. Tscheliessnig, A. Biedermann, and P. Varga, Surf. Sci. 478, L355 (2001).

[47] S. Schintke, S. Messerli, K. Morgenstern, J. Nieminen, and W.-D. Schneider, J. Chem. Phys. 114, 4206 (2001).

[48] M.-F. Hsieh, D.-S. Lin, H. Gawronski, and K. Morgenstern, J. Chem. Phys. 131, 174709 (2009).

[49] B. C. Stipe, M. A. Rezaei, W. Ho, S. Gao, M. Persson, and B. I. Lundqvist, Phys. Rev. Lett. 78, 4410 (1997).

[50] J. R. Hahn and W. Ho, J. Chem. Phys. 122, 244704 (2005).

[51] H. J. Monkhorst and J. D. Pack, Phys. Rev. B 13, 5188 (1976). 